\section{SUCCESSFUL ATRIAL DEFIBRILLATION WITH VERY-LOW-ENERGY SHOCKS BY MEANS OF TEMPORARY EPICARDIAL WIRE ELECTRODES}

Sustained atrial fibrillation is very common after cardiac surgical procedures. We hypothesized that atrial defibrillation could be accomplished consistently and safely by means of low-energy shocks delivered by temporary stainless steel wire electrodes placed at the time of the operation. Sterile pericarditis was created in five mongrel dogs $(20.9 \pm 2.1 \mathrm{~kg})$, and pairs of standard temporary stainless steel wire electrodes were placed on the right atrial appendage, on Bachmann's bundle, and on the right ventricular apex for pacing, sensing, and recording. Temporary stainless steel wire electrodes, insulated except for the distal $6 \mathrm{~cm}$ and used to deliver defibrillation shocks, were placed adjacent to both atrial free walls and secured to the pericardium. All electrodes were brought out through the skin, and the sternotomy was closed. Dogs were tested in the conscious state on postoperative day 2. Sustained atrial fibrillation was induced by rapid atrial pacing. A customized software program was used to control the defibrillator, which delivered $R$ wave-synchronous biphasic shocks to the atria through the temporary defibrillation electrodes. The shock intensity began at 50 volts and was increased by 10 -volt increments until atrial fibrillation was terminated. Atrial fibrillation was terminated in all dogs at $112 \pm 7$ volts, with an energy of $0.42 \pm 0.07$ joule and an impedance of $67.8 \pm 4 \mathrm{ohms}$ (all values mean \pm standard error of the mean). The mean percent success for atrial defibrillation at this minimal threshold was $49 \%$. Thus at low-threshold voltages atrial fibrillation could be terminated with every other shock. A $25 \%$ increase in the minimal threshold voltage improved the conversion rate to $73 \%$ (mean energy $0.66 \pm 0.19$ joule and mean impedance of $67 \pm 3.8 \mathrm{ohms}$ ). No complications were detected with the use of the electrodes or after their removal on the seventh postoperative day. One instance of electrode migration on the right atrial free wall was detected by roentgenography, but this did not adversely affect atrial defibrillation threshold. No ventricular arrhythmias or hemodynamic complications were noted during shock delivery. We conclude that successful conversion of atrial fibrillation to sinus rhythm can be achieved consistently with shock energies below 0.5 joule delivered with temporary epicardial defibrillation wire electrodes in this canine pericarditis model. These results suggest that this approach to the management of sustained atrial tachyarrhythmias has considerable promise in the management of atrial fibrillation in patients who have had cardiac operations. (J THORAC Cardiovasc Surg 1996;111:392-8)

Brian L. Cmolik, MD, ${ }^{\mathrm{a}}$ Jose Ortiz, MD, ${ }^{\mathrm{b}}$ Gregory M. Ayers, MD, $\mathrm{PhD},{ }^{\mathrm{c}}$ Jai H. Lee MD, ${ }^{a}$ Alexander S. Geha, MD, ${ }^{a}$ and Albert L. Waldo, MD, ${ }^{b}$ Cleveland, Ohio, and Redmond, Wash.
From the Divisions of Cardiothoracic Surgery ${ }^{\mathrm{a}}$ and Cardiology, ${ }^{\mathrm{b}}$ Case Western Reserve University/University Hospitals of Cleveland, Cleveland, Ohio, and InControl Incorporated, ${ }^{\circ}$ Redmond, Wash.

Supported in part by a grant from InControl, Incorporated, Redmond, Wash.
Read at the Twentieth Annual Meeting of The Western Thoracic Surgical Association, Squaw Valley, Calif., June 22-25, 1994.

Address for reprints: Brian L. Cmolik, MD, University Hospitals of Cleveland, 11100 Euclid Ave., Cleveland, OH 44106.

Copyright (C 1996 by Mosby-Year Book, Inc.

$0022-5223 / 96 \$ 5.00+0 \quad \mathbf{1 2 / 6 / 6 8 5 6 0}$ 
$A^{t}$ trial tachyarrhythmias are the most common type of rhythm disturbance seen after cardiac surgical procedures. The prevalence of atrial arrhythmias after cardiac operations varies between $25 \%$ and $50 \%{ }^{1-4} \mathrm{~A}$ recent large series documented the prevalence of atrial arrhythmias after isolated coronary bypass operations to be more than $30 \%$, and as the complexity of the procedures increased, the prevalence of atrial rhythm disturbances rose as high as $90 \% .^{1}$ The clinical sequelae from postoperative atrial arrhythmias range from self-limited episodes of little clinical consequence to congestive heart failure causing significant patient morbidity. Morbidity from atrial tachyarrhythmias includes hemodynamic impairment from loss of atrial contribution to cardiac output, increased risk of thromboembolic stroke, and increased length of hospitalization. The prevalence of these rhythm disturbances and their impact on patient morbidity and hospital cost has prompted several studies of perioperative pharmacologic prophylaxis to prevent atrial tachyarrhythmias from developing during the postoperative period. ${ }^{5-7}$

Management of atrial arrhythmias has included the correction of underlying electrolyte abnormalities, pharmacologic therapy, rapid atrial pacing, and direct-current cardioversion. Pharmacologic therapy has centered around the use of medications for both ventricular rate control and cardioversion. Directcurrent cardioversion has traditionally required the use of anesthetics to provide adequate pain control because of the high energy (50 to 360 joules) required for transthoracic cardioversion. Rapid atrial pacing through temporary epicardial wire electrodes is used to treat classic (type I) atrial flutter and some forms of atrial tachycardia, but it has no role in the treatment of atrial fibrillation or type II atrial flutter. ${ }^{8}$

Temporary epicardial stainless steel wire electrodes have long been used to diagnose and treat a variety of rhythm disturbances after cardiac surgical procedures. The use of temporary wire electrodes for these purposes was initially reported in the $1960 \mathrm{~s}$ by Harris and associates. 9,10 The clinical utility of temporary electrodes in both the atrial and ventricular positions has been documented. $8,11,12$ This pilot study was designed to investigate extending the role of temporary stainless steel wire electrodes for the purpose of defibrillating the atria with lowenergy shocks. We hypothesized that effective atrial defibrillation could be accomplished safely and reliably with very-low-energy shocks delivered through temporary wire electrodes placed adjacent to the left and right atria. The previously reported success of atrial defibrillation achieved with low-energy shocks delivered by endocardial electrodes placed in the coronary sinus and right atrial appendage in animal models encouraged us to pursue this hypothesis. $^{13-16}$

\section{Materials and methods}

All animals used in this study received humane care in compliance with the "Principles of Laboratory Animal Care" formulated by the National Society for Medical Research and the "Guide for the Care and Use of Laboratory Animals" prepared by the Institute of Laboratory Animal Resources and published by the National Institutes of Health (NIH Publication No. 86-23, revised 1985).

Operative technique. Five mongrel dogs weighing an average of $20.9 \mathrm{~kg}$ were studied. General anesthesia was induced with thiopental $(30 \mathrm{mg} / \mathrm{kg}$ ) and maintained with $1 \%$ halothane and nitrous oxide delivered through an endotracheal tube. A median sternotomy was accomplished with an oscillating saw, and a pericardial cradle was created. Pairs of stainless steel temporary wire electrodes (0-Flexon, Davis \& Geck, Danbury, Conn.) coated with Teflon polytetrafluoroethylene were placed on the right atrial appendage, Bachmann's bundle, and at the right ventricular apex for pacing, sensing, and recording. All electrodes were brought out through the skin through separate stab wounds on the flanks of the animals and were secured with nonabsorbable suture. Custom-made defibrillation wire electrodes with an outer diameter of 0.016 inch and insulated except for the distal $6 \mathrm{~cm}$ (InControl, Incorporated, Redmond, Wash.) (Fig. 1) were woven into the pericardium with the exposed wire adjacent to the atrial epicardial surface. The right atrial electrode was secured parallel to the course of the superior and inferior venae cavae along the right atrial free wall, and the left atrial electrode was secured parallel and superior to the course of the coronary sinus. Thus the bulk of the atrial muscle mass was between the two exposed surfaces of the wire electrodes. Electrode position is demonstrated in Fig. 2. Each of these electrodes was also externalized through a stab wound in the flank and secured with nonabsorbable suture. Right and left atrial surfaces were then dusted with sterile talcum powder in the manner described by Pagè and associates, ${ }^{17}$ with care taken not to apply the powder to the surfaces of the ventricles. The pericardium was then reapproximated with nonabsorbable suture, the sternotomy was closed in standard fashion, and the dog was allowed to recover. A postoperative chest x-ray film was obtained in all dogs. All animals received perioperative antibiotic administration with parenteral cefazolin $(1000 \mathrm{mg})$. Postoperative antibiotic treatment consisted of enterally administered amoxicillin-clavulanic acid ( $250 \mathrm{mg}$ twice a day). Postoperative pain was managed with subcutaneously administered buprenorphine $(0.03 \mathrm{mg}$ twice a day).

Atrial defibrillation studies. The efficacy and safety of atrial defibrillation effected with the temporary defibrilla- 


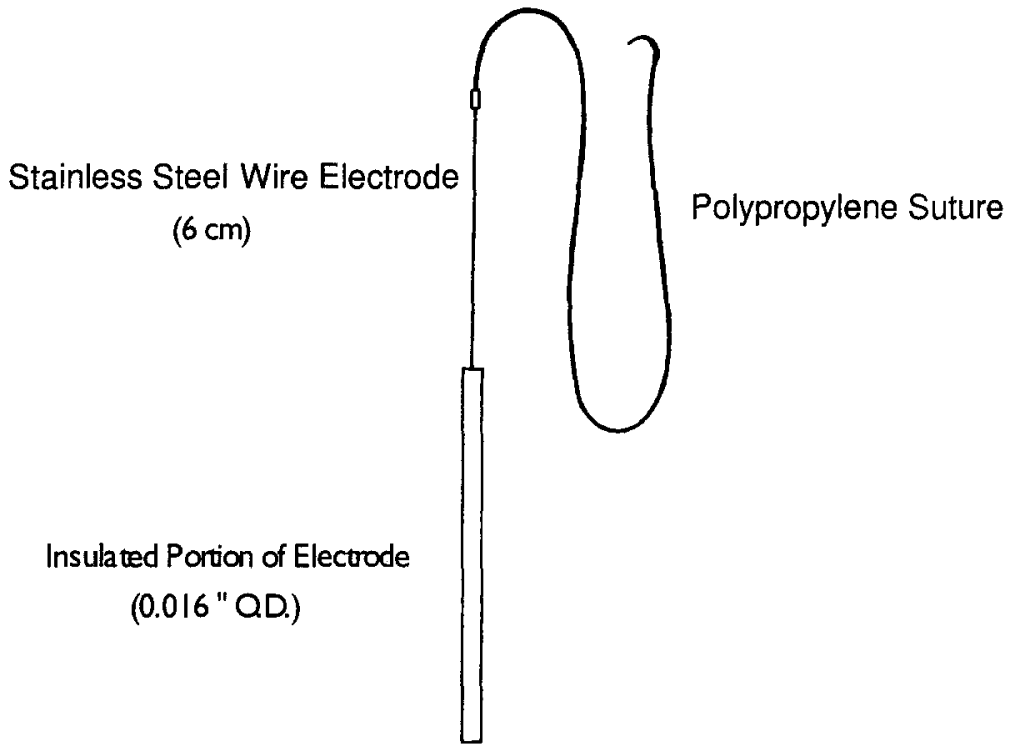

Fig. 1. Diagram of a stainless steel electrode used for atrial defibrillation. O.D., Outer diameter.

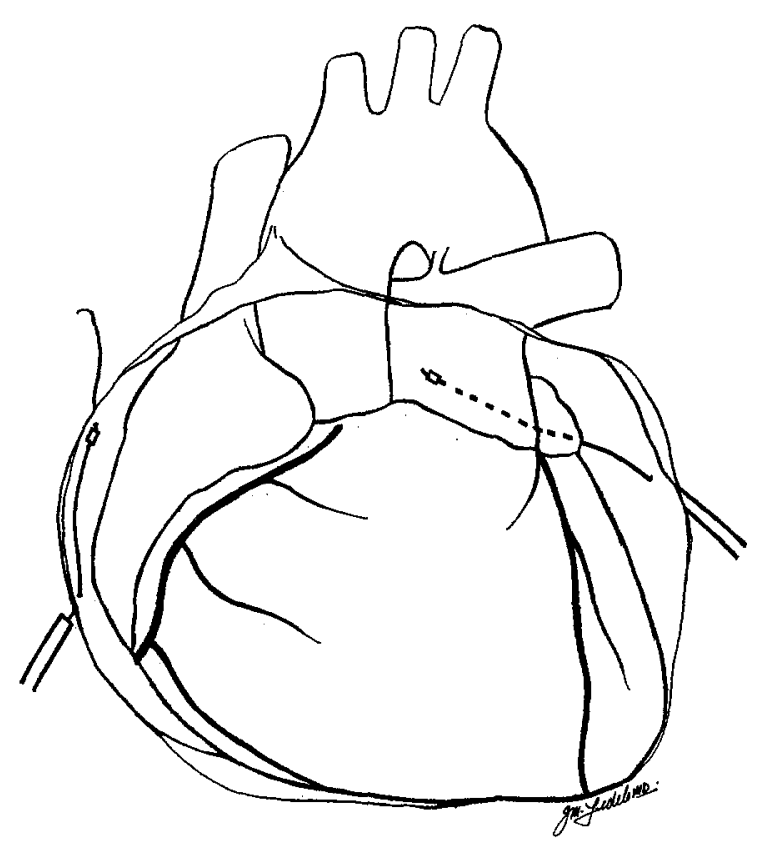

Fig. 2. Location of temporary stainless steel defibrillation electrodes in the pericardial sac when the heart is viewed anteriorly. The dotted line represents the course of the electrode running just above the level of the coronary sinus.

tion wire electrodes were evaluated on postoperative day 2. An anteroposterior chest $\mathrm{x}$-ray film was obtained to verify wire electrode positions before testing by comparing the film with one taken after the operation. The animals were tested in a conscious but sedated state (xylazine, $0.2 \mathrm{mg} / \mathrm{kg}$ ) for this portion of the study. Electrocardiogram leads I, II, and III and electrograms from the right atrial appendage, right ventricular apex, and Bachmann's bundle were monitored and recorded on a switched-beam oscilloscopic recorder (model VR-16, Electronics for Medicine, Pleasantville, N.Y.). Sustained atrial fibrillation, defined as atrial fibrillation lasting at least 5 minutes, was induced by rapid atrial pacing through the right atrial appendage electrodes. A customized software program was used to control the defibrillation (InControl), which delivered an $\mathrm{R}$ wave-synchronized biphasic shock to the atria through the bare portion of the defibrillation wire electrodes. Shock intensity began at 50 volts (known to be ineffective ${ }^{16}$ ) and was increased in 10 -volt increments until atrial fibrillation was terminated. Twenty shocks at this voltage were then tested to determined the percent of successful conversion to sinus rhythm. If the percent conversion was between $10 \%$ and $90 \%$, this value was designated the minimum threshold voltage. If the percent conversion was below $10 \%$ or above $90 \%$, the voltage was raised or lowered, respectively, and testing was repeated until the percent success fell into the latter range. Shock intensities that were approximately $25 \%$ higher than the minimum threshold voltage were then tested in a similar 20-shock protocol to determine whether percent conversion to sinus rhythm would increase.

On the seventh postoperative day, the defibrillation wire electrodes were removed from all dogs while they were under a general anesthetic. During this time, cardiac rhythm was monitored continuously with an electrocardiogram in all animals and blood pressure with an indwelling femoral artery catheter connected to a strain gauge and amplifier. The animals were observed for signs of hemodynamic instability or arrhythmias for half an hour after the defibrillation wire electrodes were removed, and then they were put to death. All hearts were excised and 


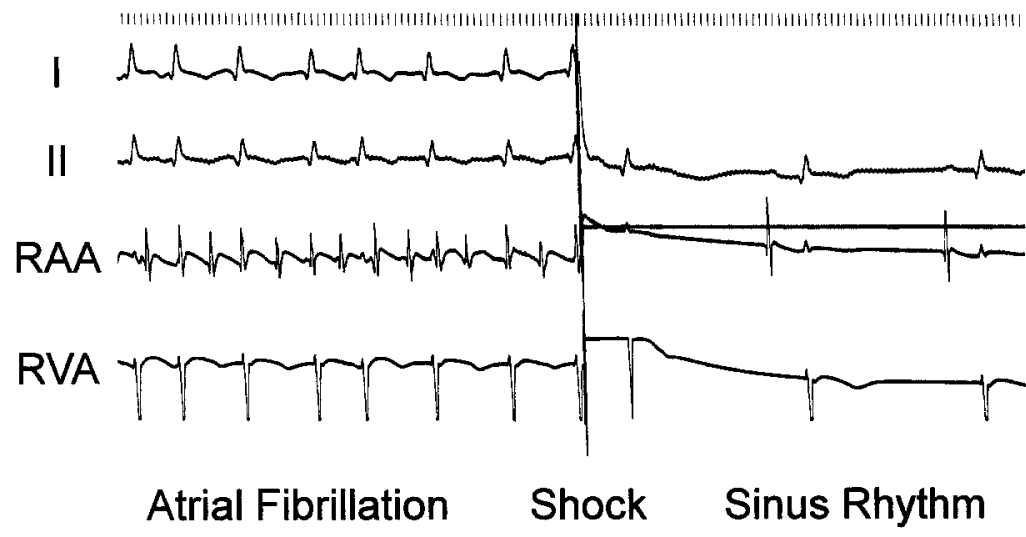

Fig. 3. An example of atrial defibrillation in the canine model of sterile pericarditis with stainless steel temporary atrial defibrillation wires. $R A A$, Right atrial appendage; $R V A$, right ventricular apex.

inspected for evidence of trauma related to the defibrillation wire electrodes.

\section{Results}

After sustained atrial fibrillation was induced, the atria were successfully defibrillated in each of the five dogs. A representative example is shown in Fig. 3. Migration of a defibrillation wire electrode was noted in one animal, probably because of improper weaving of the wire into the pericardium. In this animal, only the studies to determine the minimum threshold voltage were performed. The animal was then put to death after the minimum threshold voltage had been determined, and the pericardium was inspected for wire electrode migration. Both defibrillator wire electrodes had been displaced to a minimal extent, but atrial defibrillation had been successfully accomplished nonetheless.

No evidence of trauma from the multiple defibrillations was noted on either the right or left atrium after the hearts were excised. All sets of defibrillating wire electrodes were removed easily and without complication by simply applying gentle traction at the insertion site in the same manner that is used to remove a temporary cardiac pacing wire. Hemodynamic criteria indicated no evidence of bleeding, and no bleeding was detected when the chest was inspected after the animal was put to death.

Atrial defibrillation data. Tables I and II describe the values obtained at the minimum threshold voltage and after an increase in the minimum mean threshold voltage of approximately $25 \%$, as tested, to determine the percent of successful conversion from atrial fibrillation to normal sinus rhythm in each study. The animals were tested in the conscious
Table I. Values obtained at the minimum threshold voltage (MTV) for atrial fibrillation

\begin{tabular}{ccccc}
\hline Animal No. & $\begin{array}{c}\text { MTV } \\
\text { (volts) }\end{array}$ & $\begin{array}{c}\text { Impedance } \\
\text { (ohms) }\end{array}$ & $\begin{array}{c}\text { Energy } \\
\text { (joule) }\end{array}$ & $\begin{array}{c}\text { Conversion to } \\
\text { sinus rhythm } \\
(\%)\end{array}$ \\
\hline 1 & 100 & 67 & 0.33 & 45 \\
2 & 110 & 71 & 0.38 & 35 \\
3 & 110 & 58 & 0.41 & 75 \\
4 & 140 & 62 & 0.67 & 40 \\
5 & 100 & 85 & 0.30 & 50 \\
Mean \pm SEM & $112 \pm 7$ & $68 \pm 4$ & $0.42 \pm 0.07$ & 49
\end{tabular}

$S E M$, Standard error of the mean.

state and tolerated the shock deliveries well. Each animal was tested a minimum of 40 times between evaluation of the minimum threshold voltage and a $25 \%$ increase in the minimum threshold voltage. Intravenous sedation was adequate to control any potential discomfort. At the mean minimum threshold voltage of $112 \pm 7$ volts, the mean energy needed to convert atrial fibrillation to sinus rhythm was $0.42 \pm 0.07$ joule, the mean impedance was $68 \pm 4$ ohms, and the percent of successful conversion to sinus rhythm at this mean shock energy was $49 \%$. When the mean minimum threshold voltage was increased by $25 \%$ to a mean of $140 \pm 17$ volts, the mean shock energy increased to $0.66 \pm 0.19$ joule, but the mean impedance remained approximately the same at $67 \pm 4$ ohms. However, the rate of conversion increased to $73 \%$.

\section{Discussion}

This study demonstrates that in the model of sterile pericarditis with atrial fibrillation, successful atrial defibrillation could be safely achieved at very 
Table II. Values obtained at an increase of approximately $25 \%$ in the minimum threshold voltage (MTV)

\begin{tabular}{ccccc}
\hline Animal No. & $\begin{array}{c}\text { MTV } \\
\text { (volts) }\end{array}$ & $\begin{array}{c}\text { Impedance } \\
\text { (ohms) }\end{array}$ & $\begin{array}{c}\text { Energy } \\
\text { (joule) }\end{array}$ & $\begin{array}{c}\text { Conversion } \\
\text { to sinus } \\
\text { rhythm } \\
(\%)\end{array}$ \\
\hline 1 & 120 & 66 & 0.48 & 85 \\
2 & Wire migration & - & - & - \\
3 & 130 & 60 & 0.50 & 55 \\
4 & 190 & 64 & 1.24 & 85 \\
5 & 120 & 78 & 0.44 & 65 \\
Mean \pm SEM & $140 \pm 17$ & $67 \pm 4$ & $0.66 \pm 0.04$ & 73 \\
\hline
\end{tabular}

$S E M$, Standard error of the mean.

low energy ( 0.30 to 0.67 joule) and in reproducible fashion by means of temporary defibrillation wire electrodes. This observation is consistent with atrial defibrillation data from the same sterile pericarditis model with electrode catheters placed in the coronary sinus and right atrial appendage. ${ }^{16}$ Furthermore, the choice of the sterile pericarditis model for study is important because pericarditis is believed to be one of the important factors that predisposes the human atria to fibrillate after heart surgical procedures. ${ }^{8,17}$ Thus this model would appear to represent a close clinical counterpart for postoperative atrial fibrillation in human beings in terms of both arrhythmia mechanism and time course ${ }^{18}$ and a logical choice for the evaluation of therapies for postoperative atrial fibrillation.

The atrial defibrillation threshold. Unlike ventricular tachyarrhythmias, atrial tachyarrhythmias are generally well tolerated for short periods. Thus, whereas the need for immediate defibrillation is obvious for patients with ventricular fibrillation, immediate restoration of sinus rhythm for patients who have atrial fibrillation after cardiac operations is rarely necessary. This clinical principle governed the selection of the minimum threshold voltage, because it was not necessary that each shock be successful, but that an acceptable conversion rate at low-shock energy be achieved. This principle also recognized that atrial fibrillation early after cardiac operations (within 2 to 3 days of the operation) may be successfully converted, but because atrial fibrillation is likely to recur, simply controlling the ventricular response rate may initially be the appropriate therapy. Nevertheless, ultimate restoration of sinus rhythm is necessary to avoid the short-term and long-term sequelae associated with this rhythm disturbance. Especially because atrial defibrillation is elective, defibrillation with epicardial defibrillation wire electrodes should be reliable, safe, and well tolerated. In our study, because high-energy shocks were not used, the percent of successful defibrillations was well below $100 \%$. However, even at very low energy (mean $<0.5$ joule), each episode of atrial fibrillation was stopped with virtually every other shock ( $49 \%$ converted to sinus rhythm). Because immediate defibrillation is not required for the management of atrial fibrillation, the goal of safe, reliable, and well-tolerated defibrillation was met. Whether this would be true in patients is yet to be demonstrated.

Clinical implications. This pilot study demonstrates the potential utility of temporary epicardial defibrillation wire electrodes in the management of atrial fibrillation after cardiac operations in patients. Temporary epicardial wire electrodes placed at the time of cardiac surgical procedures and used in both the atrial and ventricular positions for diagnosis of postoperative arrhythmias is routine in most cardiac surgery centers. Using epicardial wires for atrial defibrillation is an extension of their widely recognized utility. Because a significant portion of patients are at risk for the development of atrial fibrillation after various cardiac surgical procedures, they should benefit from placement of defibrillation wire electrodes during the operation if low-energy atrial defibrillation can be demonstrated to be effective, safe, and tolerable. Although low-energy shocks have successfully defibrillated the atria in several animal models, ${ }^{13-16,19}$ it is not yet clear that shock energies as low as 0.5 joule, which have been successful in the animal studies, will be successful in patients.

To date, studies in patients with catheter electrodes placed in the coronary sinus and the right atrium have shown that atrial fibrillation can be successfully converted to sinus rhythm with lowenergy shocks, but these shocks have ranged from 0.5 to 2 joules or even more. ${ }^{20-23}$ Because it is likely that shocks as low as 1 joule will be felt as unpleasant in patients, it is uncertain whether this procedure will be tolerated and, therefore, accepted in clinical practice. However, it may be that temporary defibrillation wire electrodes, when used in patients to defibrillate the atria after heart surgery, may permit successful defibrillation with less than 1 joule. We suggest that further study of temporary epicardial atrial defibrillation electrodes is indicated to examine their utility in patients. The population undergoing cardiac surgical procedures is at a significant risk for postoperative atrial fibrillation and 
could benefit considerably from this therapy in the postoperative period.

\section{REFERENCES}

1. Creswell LL, Schuessler RB, Rosenbloom M, Cox JL. Hazards of postoperative atrial fibrillation. Ann Thorac Surg 1993;56:539-49.

2. Michelson EL, Morganroth J, MacVaugh H. Postoperative arrhythmias after coronary artery and cardiac valvular surgery detected by long-term electrocardiographic monitoring. Am Heart J 1979;97:442-8.

3. Khuri SF, Okike N, Joas M, et al. Efficacy of Nadolol in preventing supraventricular tachyarrhythmia after coronary bypass grafting. Am J Cardiol 1987;60:51D.

4. Yousif H, Davies G, Oakely CM. Peri-operative supraventricular arrhythmias in coronary bypass surgery. Int J Cardiol 1990;26:313-8.

5. Mills SA, Pool GV Jr, Breyer RH, et al. Digoxin and propranolol in the prophylaxis of dysrhythmias after coronary bypass grafting. Circulation 1983;68(Suppl): III222-5.

6. Silberman NA, Wright R, Levitsky S. Efficacy of low-dose propranolol in preventing postoperative supraventricular tachyarrhythmias: a prospective, randomized study. Ann Surg 1982;196:194-7.

7. White HD, Antman EM, Glynn MA, et al. Efficacy and safety of timolol for prevention of supraventricular tachyarrhythmias after coronary bypass surgery. Circulation 1984;70:479-84.

8. Waldo AL, MacLean WAH. Diagnosis and treatment of cardiac arrhythmias following open heart surgery. Mount Kisco, New York: Futura, 1980:115-29.

9. Harris PD, Singer DH, Malm JR, Hoffman BF. Chronically implanted cardiac electrodes for diagnosis, therapeutic, and investigational use in man. J Thorac Cardiovasc Surg 1967;54:191-8.

10. Harris PD, Malm JR, Bowman FO Jr, Hoffman BF, Kaiser GA, Singer DH. Epicardial pacing to control arrhythmias following cardiac surgery. Circulation 1968;37(Suppl):II178-83.

11. Waldo AL. Arrhythmias following open heart surgery: role of cardiac pacing and recording. In: Grillo HC, Austen WG, Wilkens EW, Mathisen DJ, Vlahakes GJ, eds. Current therapy in cardiothoracic surgery. Toronto: BC Decker Inc., 1989:289-92.

12. Waldo AL, MacLean WAH, Cooper TB, Kouchoukos NT, Karp RB. The use of temporarily placed epicardial atrial wire electrodes for the diagnosis and treatment of cardiac arrhythmias following open heart surgery. J Thorac Cardiovasc Surg 1978;76:500-5.

13. Cooper RAS, Alferness CA, Smith WM, Ideker RE. Internal cardioversion of atrial fibrillation in sheep. Circulation. 1993;87:1673-86.

14. Powell A, Garan H, McGovern B, et al. Low energy conversion of atrial fibrillation in the sheep. J Am Coll Cardiol 1992;20:707-11.
15. Ayers GM, Ilina MI, Wagner DO, Sirokman WA, Griffin JC, Alferness CA. Right atrial electrode location for transvenous atrial defibrillation. J Am Coll Cardiol 1994;23:125A.

16. Ortiz J, Niwano S, Abe H, Gonzalez X, Ayers GM, Waldo AL. Transvenous atrial defibrillation in two canine models of atrial fibrillation. J Am Coll Cardiol 1994;23:125A.

17. Pagè PL, Plumb VJ, Okumura K, Waldo AL. A new animal model of atrial flutter. J Am Coll Cardiol 1986;8:872-9.

18. Ortiz J, Igarashi M, Gonzalez X, Johnson NJ, Waldo AL. A new, reliable atrial fibrillation model with a clinical counterpart. J Am Coll Cardiol 1993;21:183A.

19. Ayers GM, Griffin JC, Ilina MB, et al. An implantable atrial defibrillator: initial animal experience with novel device [Abstract]. PACE 1994;17:769.

20. Keane D, Sulke N, Cooke R, et al. Endocardial cardioversion of atrial flutter and fibrillation [Abstract]. PACE 1993;16:928.

21. Johnson EE, Yarger MD, Wharton JM. Monophasic and biphasic waveforms for low energy internal cardioversion of atrial fibrillation in humans [Abstract]. Circulation 1993;88(Suppl):I59.

22. Murgatroyd FD, Johnson EE, Cooper RA, et al. Safety of low energy transvenous atrial defibrillation-worldwide experience [Abstract]. Circulation 1994;90(Suppl):I14.

23. Brady GH, Hoferis B, Johnson G, et al. Implantable transvenous cardiovert-defibrillators. Circulation 1993;87:1152-68.

24. Alt R, Schmitt C, Ammer R, et al. Initial experience with intracardiac atrial defibrillation in patients with chronic atrial fibrillation. PACE 1994;1067-78.

\section{Discussion}

Dr. Leigh I. G. Iverson (Oakland, Calif.). Atrial fibrillation is a common and often distressing arrhythmia with potential consequences involving control of the rate, possible thromboembolic phenomena, and the loss of ventricular augmentation by atrial systole. Among patients having cardiac surgery, $25 \%$ to $35 \%$ have atrial fibrillation after the operation. In the general medical population it is the most common cause of hospital admission for an arrhythmia. Almost one third of these patients will require long-term anticoagulation and approximately one fourth will require electrical conversion because of failure of medication to achieve a more physiologic rhythm. Clearly the patient population who can benefit from the resumption of a normally conducted atrial beat is large. Therefore, the implications of this study have a far greater impact than an interesting protocol involving treating a group of dogs with induced atrial fibrillation.

I have a number of comments regarding this study. First, the number of dogs is very small, and it is difficult to base conclusions on small numbers. Dog atria are smaller than normal human atria, and in this protocol they are presumably normal. None of these dogs had atriotomies, 
hypothermia, or cardiopulmonary bypass with the unpredictable fluid shifts and the variable ischemia associated with different methods of cardioplegia. Furthermore, no comparison is made to the physiologic conditions seen in patients who have chronic valvular disease. Atrial fibrillation was induced on multiple occasions in these dogs; clearly the treatment of acute atrial fibrillation is different from the treatment of chronic atrial fibrillation. The arrhythmia lasted for seconds or perhaps minutes before conversion. We deal with a patient population that may have an arrhythmia that is present for hours, perhaps days, and sometimes for several weeks. The substrate in the human population, therefore, is totally different from the one that you described in this experimental model. How do you think the information you have obtained transfers to human beings who are in a completely different physiologic and electrophysiologic situation?

Dr. Cmolik. In terms of the substrate and the mechanism, one of the important features of the model is that it uses pericarditis to induce atrial fibrillation. Most electrophysiologists would agree that this is probably the common denominator in patients having atrial fibrillation. The mechanism for the development of atrial fibrillation in these dogs is a reentry phenomenon. I cannot say that it is almost completely identical to that in the human heart, because the human atrium has not been completely mapped. However, the atrial fibrillation seems to start in the lateral wall, and it is a reentry phenomenon that is very similar to that in the human heart. In terms of the mechanism, we think this model is very appropriate.

I agree that the substrate may be different. The dogs were not subjected to bypass, they did not have atriotomy, and there was no difference in terms of temperature. However, the two species are very similar in terms of the mechanism for the development of atrial fibrillation and the defibrillation characteristics.

Dr. Iverson. Dog 2 disappears in your second set of data. You said that the atrial leads migrated in this dog. You discuss no proarrhythmic potential for these leads, but I wonder if that is the reason that the second dog was excluded from further study. Other studies, particularly in sheep, show a potential for ventricular arrhythmias on atrial defibrillation in $0.5 \%$ to $0.7 \%$ of subjects.

You said that the dogs were conscious but that no dog was sedated to the point that it required intubation. In human beings defibrillation causes pain, even at the low number of joules often required to convert atrial fibrillation. I am unclear both on how much pain control was required and on the issue of the proarrhythmic effects of defibrillation in these dogs.

Dr. Cmolik. In $\operatorname{dog} 2$ the right atrial electrode migrated inferiorly. We conducted minimum threshold voltage determinations in this dog, but we were interested to see why the had wire migrated. We subjected the dog to general endotracheal anesthesia, opened the sternotomy, and examined the dog. We modified our wire protocol insertion technique at that time. Instead of one stitch through the pericardium with Prolene suture (Ethicon, Inc., Somerville, N.J.), we weaved it in with two stitches, and it had nothing to do with a proarrhythmic effect.
The proarrhythmic effect at atrial defibrillation was reported by one of the coauthors, Dr. Ayers, in Circulation recently, and the study was done to see exactly what a safe interval is so as not to produce the " $\mathrm{R}$ on $\mathrm{T}$ phenomenon." In dogs, an RR interval of more than $280 \mathrm{msec}$ is generally safe. Most of our dogs were between 300 and $400 \mathrm{msec}$ in terms of sensing the RR interval and avoiding an $\mathrm{R}$ on $\mathrm{T}$ phenomenon. In more than 1000 experiments in similar dogs, with catheter-induced atrial arrhythmias, thoracotomy approaches, and median sternotomies, we have had no incidents of ventricular arrhythmias in this model based on using a longer RR interval to sense when to shock on the $\mathrm{R}$ wave.

Dr. Iverson. Despite these issues, I think it is an excellent study. It has demonstrated that low-energy defibrillation of 0.4 to 0.7 joule delivered in a biphasic waveform by means of removable electrodes appears safe and effective. It would suggest that human studies are warranted, particularly for the patient with an acute fibrillation after a cardiac operation. It raises the question whether this is a potential treatment modality for patients who have paroxysmal atrial fibrillation unrelated to the operation. This brings up a whole series of issues of arrhythmia recognition, implantability, pain control, and recognition of who is a candidate. I am concerned that we do not have adequate data at this point to discuss the potential incidence of inducing ventricular tachycardia or fibrillation with electroconversion of atrial fibrillation.

Your paper raises a number of issues that can be resolved only by further investigation, including an investigation in primates and probably in human subjects. Have you done any further studies along this line? In particular, have you attempted to use this technique in patients who have acute atrial fibrillation during the postoperative course?

Dr. Cmolik. This is currently being submitted before our institutional review board. We are going to do further studies that involve the use of cardiopulmonary bypass. We also plan to use the wires to defibrillate atrial flutter and rapid atrial pace out of atrial flutter and for general pacing in the postoperative period. I think the utility of these wires stems from the fact that they can be used for routine pacing in patients with bradycardia. I hope they can be used for atrial defibrillation and to convert both atrial fibrillation and atrial flutter.

Regarding the pain issue, the dogs were sedated to keep them from wandering around the laboratory more than for pain control. Studies in England have shown that people start to perceive shocks at about 0.5 joule. At our minimum threshold voltage we were about $20 \%$ below that. The whole goal is for this to be a relatively well-tolerated procedure. The rhythm is completely different from ventricular fibrillation, in which it is necessary to restore some sort of hemodynamic stability and conduction immediately to that patient. Even if the conversion rate is only $10 \%$, if the procedure is well tolerated, there is time to convert the arrhythmia using very-low-energy shocks. That is the goal of using this type of wire. 